\title{
MATrix LABoratory Algorithm in Ultrasound Image-Guided General Drug Anesthesia along with Lumbar and Sacral Plexus Block in Hip Replacement under
}

\author{
Nana Wang $(\mathbb{D}$, Lingyang Chen $(\mathbb{D}$, Hongzhu Wang $(\mathbb{D})$, Yibing Wang $(\mathbb{D}$, and Binhao Ruan $(\mathbb{D})$ \\ Department of Anesthesiology, Taizhou Hospital of Zhejiang Province affiliated to Wenzhou Medical University, Taizhou 317000, \\ Zhejiang, China \\ Correspondence should be addressed to Binhao Ruan; yuanbh@enzemed.com
}

Received 28 July 2021; Revised 15 September 2021; Accepted 17 September 2021; Published 8 October 2021

Academic Editor: Gustavo Ramirez

Copyright (c) 2021 Nana Wang et al. This is an open access article distributed under the Creative Commons Attribution License, which permits unrestricted use, distribution, and reproduction in any medium, provided the original work is properly cited.

Objective. The study aimed to explore the application of ultrasound image-guided general drug anesthesia combined with lumbar and sacral plexus block based on MATrix LABoratory (MATLAB) algorithm in hip arthroplasty and to study its clinical effect. Methods. The classic geodesic active contour (GAC) algorithm and the improved fuzzy clustering level set algorithm were used to segment ultrasound images of waist plexus, and then their segmentation effects were compared. Both algorithms are from the MATrix LABoratory (MATLAB) platform. A total of 60 patients undergoing hip arthroplasty were selected and randomly enrolled into control and experimental groups. The control group accepted general drug anesthesia, and the experimental group accepted ultrasound-guided lumbar and sacral plexus block combined with general anesthesia. The mean arterial pressure and heart rate at $\mathrm{t} 0$ (before anesthesia), $\mathrm{t} 1$ (before ventilation), $\mathrm{t} 2$ (when the skin was incised), $\mathrm{t} 3$ (when the prosthesis was implanted), $\mathrm{t} 4$ (when the incision was closed), and $\mathrm{t} 5$ (at the end of ventilation) were observed, and the intraoperative sufentanil dosage and $24 \mathrm{~h}$ analgesic dosage, the incidence of postoperative delirium, and the incidence of cognitive dysfunction were recorded. Results. The improved fuzzy clustering level set algorithm was better than the GAC model algorithm in image segmentation and running time. In contrast with the control group, the average arterial pressure and heart rate of the experimental group at the four time points of t1, $\mathrm{t} 2, \mathrm{t} 3$, and $\mathrm{t} 5$ were obviously reduced $(P<0.05)$. In contrast with the control group, the amount of sufentanil and analgesics in the experimental group was obviously reduced $(P<0.05)$, and the incidence of postoperative cognitive dysfunction and delirium was obviously reduced $(P<0.05)$. Conclusion. The improved fuzzy clustering level set algorithm is superior to the GAC model in image segmentation and running time. Under its guidance, the lumbar and sacral plexus block combined with general anesthesia has a good clinical effect in hip arthroplasty, which is better than simple general anesthesia.

\section{Introduction}

Hip fracture is a frequently occurring disease in clinic. The clinical manifestations of patients are swelling of the limbs, restricted movement, and pain, which are more common in elderly patients, often accompanied by the decline of various organs [1]. At present, the main method to treat this disease is total hip arthroplasty (THA). It can effectively improve hip joint function and reduce intraoperative bleeding, with less complications. In hip replacement surgery, general anesthesia is often required. However, most patients with hip fracture are the elderly, who are accompanied by various chronic diseases, low immunity, poor liver and kidney function [2-4], high blood loss, obvious stress response, and more sensitivity to anesthetics. Low-dose anesthetics can affect respiratory and circulatory functions in patients, and large-dose anesthesia is intolerable [5-7]. Lumbar plexus block (LPB) is currently the most commonly used anesthesia method for THA. Compared with traditional simple general anesthesia and intraspinal anesthesia, lumbar block combined with general anesthesia not only facilitates respiratory management but also effectively reduces circulatory depression. Lumbar plexus block combined with general anesthesia can be widely used in lower extremity surgery 
including marrow joint replacement surgery. Ultrasoundguided nerve block can directly block the peripheral nerves under direct vision and enable clinicians to observe the structure of the nerve and dynamically observe the spread of local anesthetics, which improves the success rate and reduces complications, laying the foundation for the successful puncture block $[8,9]$.

Ultrasound is convenient, reproductive, nondestructive, and real time and thus commonly used in orthopedics of various hospitals. In ultrasound imaging technology, the diffraction and reflection characteristics of ultrasound are used to inject ultrasound into the human body. The echo signals received according to the different impedance and attenuation coefficients of the tissue are different. These signals will be recorded in the ultrasound detector to reflect the changes in the position and intensity of the organs, and then whether the examined organs have lesions is detected [10-12]. Ultrasound equipment needs to be combined with an ultrasound imaging system to improve the diagnostic efficiency of the ultrasound department. At the same time, to better manage ultrasound imaging information, the ultrasound imaging system needs to be continuously optimized [13-15]. Image segmentation occupies an important position in the quantitative and qualitative analysis of medical ultrasound images, which directly affects the subsequent analysis and processing [16]. Correct and clear ultrasound image segmentation provides a guarantee for the accurate extraction of diagnostic information in clinical applications, and it is also the key to clinical quantitative analysis, realtime monitoring, and precise positioning of computer-aided operations $[17,18]$. In recent years, with the advancement of segmentation technology, technologies such as dynamic programming, active contour models, and level sets have made image segmentation develop rapidly from two-dimensional segmentation to three-dimensional segmentation, which continuously improves image segmentation [19-21]. Traditional ultrasound image segmentation is mainly based on the edge detection method and the region growing method, which complement each other and need to be combined in clinical practical applications [22]. Fuzzy clustering level set algorithm is a model that has been studied more in the field of image analysis and computer vision, but its application in ultrasound is limited due to the characteristics of large ultrasound noise [23]. Therefore, in recent years, many experts and scholars have devoted themselves to adapting the fuzzy clustering level set algorithm to the processing of ultrasound images, and certain progress has been made, such as the proposal of a binarization algorithm based on the fuzzy clustering level set algorithm. However, there are still some problems in related research. If this algorithm is to be widely used in ultrasound image processing, further research is needed [24]. MATLAB is currently one of the most useful software applications in the world, and it has powerful computing and visualization capabilities. It has more than 30 toolboxes for different areas. It is the preferred tool in the field of computer-aided design and algorithm simulation [25].

Therefore, in this study, MATLAB software was used to improve the fuzzy clustering level set algorithm, which was then applied to ultrasound image-guided general anesthesia combined with lumbosacral plexus block in hip arthroplasty, to provide a scientific basis for improving the efficiency of ultrasound diagnosis and auxiliary diagnosis and promote the rehabilitation of hip replacement.

\section{Materials and Methods}

2.1. Research Subjects. A total of 60 patients undergoing hip arthroplasty in the hospital from March 2019 to March 2020 were selected, and 30 of them were enrolled into a control group and the remaining were enrolled into an experimental group. The control group had 17 males and 13 females, with an average age of $(51.2 \pm 3.7)$ years, there were 16 males and 14 females in the experimental group, with an average age of $(52.8 \pm 2.4)$ years, and there was no obvious difference in general information. Exclusion criteria were as follows: patients with severe cardiovascular and cerebrovascular diseases and lung infections during the perioperative period and those who were not suitable for general anesthesia; those with abnormal coagulation function; those with a history of mental illness; those who were allergic to anesthetics; and those not cooperating or refusing lumbar nerve block. The experiment met the requirements of medical ethics, and all patients were informed of it and signed the consent form.

\subsection{Ultrasonic Image Segmentation Algorithm Based on} $M A T L A B$. Ultrasound image segmentation adopts the level set active contour model (LSACM) energy function structure, which mainly includes evolution curve and image to be segmented. The model in which the evolution curve adopts the implicit function expression is called the geometric active contour model, which introduces the level set algorithm and can naturally adapt to the change of the curve topology. The GAC model is a classic representative of the geometric active contour model. The energy functional function of the GAC model is

$$
L_{-} R(F(s))=\int{ }_{-} 0 \wedge L(F(s)):=y(|| \nabla I \dagger F(s) \mid) \mid \mathrm{d} s \dagger,
$$

in which $F(s)$ is the initial curve with $s$ as the parameter, and the function $y$ will be constructed according to the edge information of the image, which is defined as

$$
y(|\nabla I(a, b)|)=\frac{1}{\left(1+\left|\nabla G_{-} \partial * I(a, b)\right| \wedge 2\right)},
$$

in which $G_{\partial}$ is the Gaussian filter function with mean square $\partial, I(a, b)$ is the expression of the image, and $*$ is the convolution operator. Combining equation (1) with curve evolution theory, we can get GAC level set evolution equation as follows:

$$
\frac{(\partial \varnothing(t))}{\partial t}=g|\nabla \varnothing| \operatorname{div}\left(\frac{(\nabla \varnothing)}{|\nabla \varnothing|}\right)+c g|\nabla \varnothing|+\nabla g \cdot \nabla \varnothing
$$

Because the stability of the evolution equation is not high, Osher proposed a new level set method, introduced the Heaviside function, and constructed a new energy functional function, expressed as follows: 


$$
\begin{aligned}
\frac{(\partial \varnothing)}{\partial t}= & g \operatorname{div}\left(\frac{(\nabla \varnothing)}{|\nabla \varnothing|}\right) \delta_{-} \varepsilon(\varnothing)+\nabla g \\
& \cdot \frac{(\nabla \varnothing)}{|\nabla \varnothing|} \delta_{-} \varepsilon(\varnothing)+c g \delta_{-} \varepsilon(\varnothing),
\end{aligned}
$$

in which $\delta_{\varepsilon}(\varnothing)$ is the regular expression of $\delta(\varnothing)$.

This research was mainly based on Kumar et al.'s fuzzy level set-based image algorithm [14], and the improved level set evolution based on fuzzy clustering is expressed as

$$
\begin{aligned}
\frac{(\partial \varnothing)}{\partial t}= & \mu\left(\Delta \varnothing-\operatorname{div}\left(\frac{(\nabla \varnothing)}{|\nabla \varnothing|}\right)\right)+\gamma \delta(\varnothing) \operatorname{div}\left(\frac{g(\nabla \varnothing)}{|\nabla \varnothing|}\right) \\
& +g G\left(R_{-} k\right) \cdot \delta(\varnothing)+c g \delta_{-} \varepsilon(\varnothing) .
\end{aligned}
$$

The evolution step length is set as $s$, where $\mu=0.2 / \mathrm{s}$ and $\gamma=0.1$ s. The level set curve evolution equation is solved according to the set parameters, as shown in Figure 1.

\subsection{Evaluation of Segmentation Effects of the Two Algorithms.} The following three criteria are used to objectively evaluate the segmentation effects of multiple ultrasound images of the two algorithms.

Regional contrast is expressed as follows:

$$
C_{r}=\frac{f_{0}-f_{b}}{f_{0}+f_{b}},
$$

where $f_{0}$ and $f_{b}$ are the average gray levels of the target area and the background area, respectively. Greater area contrast $C_{r}$ indicates a greater difference between the target area and the background, and the segmentation effects are more obvious.

Regional consistency $U_{r}$ is expressed as follows:

$$
U_{r}=\frac{1-\left(\sigma_{1}+\sigma_{2}\right)}{A}
$$

where $A$ represents the total number of pixels in the entire image, and the normalization factor is used here. $\sigma_{1}$ and $\sigma_{2}$ are the variances of the two regions, respectively. Greater regional consistency $U_{r}$ indicates better similarity of the segmented images.

Maximum Shannon entropy $H_{\max }$ is expressed as follows:

$$
H_{(p)}=-P_{1} \log _{2} P_{1}-P_{0} \log _{2} P_{0},
$$

where $P_{0}$ and $P_{1}$ represent the probability that the binary output $Y_{[n]}$ of the segmented image is 0 and 1 and $H_{\max }$ represents the amount of information contained in most images (larger $H_{\max }$ of the target image after segmentation indicates better overall segmentation effects).

2.4. Simulation of Two Ultrasonic Segmentation Algorithms. The initial population of the improved fuzzy clustering level set algorithm based on MATLAB is Pop $=30, \varepsilon \mathrm{GA}=5.0$; for

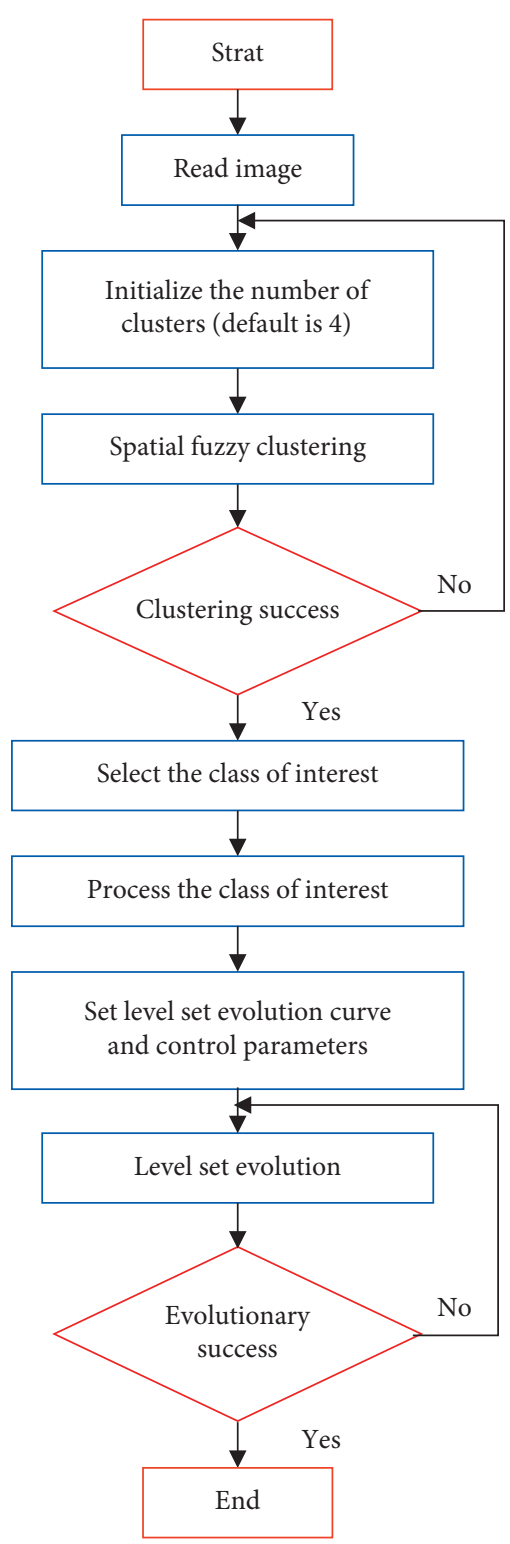

FIgUre 1: The algorithm flowchart.

the GAC model algorithm, err_goal $=0.002,1 \mathrm{r}=0.01$. The actual output TT is

$$
\begin{aligned}
& \text { TT }=0.00061 .0001-0.0001-0.00010 .003 \\
& 0.0148-0.00070 .9971-0.0056-0.0038 \\
& 0.0241-0.0009-0.00460 .9911-0.0060 \\
& 0.0283-0.0007-0.0055-0.01050 .9925
\end{aligned}
$$

The algorithm obtained is used to train the target curve, and then it is analyzed for the running time and the number of iterations.

2.5. Methods of Anesthesia. Patients in the control group were given direct general anesthesia. $0.25 \mathrm{mg} / \mathrm{kg}$ midazolam, $2.0 \mathrm{mg} / \mathrm{kg}$ propofol, $0.2 \mathrm{mg} / \mathrm{kg}$ cisatracurium bromide, and $0.4 \mu \mathrm{g} / \mathrm{kg}$ sufentanil were injected intravenously with mechanical ventilation.

The experimental group received lumbar plexus block on the affected side before general anesthesia induction. 
The specific method was as follows. Patients in the experimental group accepted ultrasound-guided lumbar and sacral plexus block based on the improved fuzzy clustering level set image segmentation combined with general anesthesia. A low-frequency ultrasound probe was used to scan the intersection of the highest point and the midline of the iliac spine on both sides, and the lumbar plexus nerve was exposed, which was a hyperechoic oval image. After the target area was determined, based on the image segmentation under improved fuzzy clustering level set, the imaging ability was optimized, the appropriate depth of field, focus range, and gain were selected, and $3 \mathrm{~mL} \mathrm{1 \%}$ lidocaine skin infiltration anesthesia was used. Peripheral nerve plexus stimulation needle was adopted to puncture the needle. After the needle tip reached the target area, no blood was drawn back and $20 \mathrm{~mL} 0.5 \%$ ropivacaine was injected. In the same way, ultrasound-guided sacral plexus nerve block based on the improved fuzzy clustering level set image segmentation was performed. The probe was positioned at $1 / 2$ of the connection between the posterior superior iliac spine and the greater trochanter, which was a continuous hyperechoic shadow. The image of sacral plexus was a hyperechoic elliptical image between the sacrum and the ilium, and $15 \mathrm{~mL}$ of $0.5 \%$ ropivacaine was injected with the same method as described above. The patient was observed, and if there was no abnormality, mechanical ventilation and general anesthesia were given, propofol $2.0 \mathrm{mg} / \mathrm{kg}$ infusion, sufentanil $0.1 \mathrm{mg} / \mathrm{kg}$ infusion.

The induction of anesthesia before operation, maintenance of anesthesia during operation, and recovery from anesthesia after operation were the same in the two groups.

2.6. Observation Indicators. The average arterial pressure and heart rate at 6 time points were observed, which were t 0 , $\mathrm{t} 1, \mathrm{t} 2, \mathrm{t} 3, \mathrm{t} 4$, and $\mathrm{t} 5$. The amount of sufentanil used during the operation, the amount of $24 \mathrm{~h}$ analgesics, the incidence of postoperative delirium, and the incidence of cognitive dysfunction were recorded.

2.7. Statistical Analysis. The mean \pm standard deviation was adopted to express measurement data, and the comparison between the two groups was realized by the $t$-test. The enumeration data were represented by $n(\%)$, and the comparison between the two groups was achieved by the $\chi^{2}$ test. All data were processed using SPSS22.0, and a bar chart was drawn with Excel 2019. $P<0.05$ indicated that the difference was obvious.

\section{Results}

3.1. The Segmentation Results of GAC Algorithm. It was evident from Figure 2 that the GAC model can accurately segment the boundary between the L2 transverse process and the L3 transverse process, making the lumbar plexus more accurate. However, it failed to accurately segment the boundary of the waist plexus in the end. It may be because the GAC model used gradient information to construct the evolution equation of the curve. However, the edge of the ultrasound image was weak and the noise was large, and it was difficult to make the curve to the boundary more accurately.

\subsection{Segmentation Results of Improved Fuzzy Clustering Level} Set. Based on MATLAB simulation, the clustering results showed that it was close to the edge of the target area, but due to the characteristics of uneven ultrasound image and large noise, some noises also appeared in the nontarget area, as shown in Figure 3. The above clustering results after level set evolution suggested that the smaller area can be filtered out to make the edge of the target area clearer. Therefore, the algorithm based on improved fuzzy clustering level set was better than the algorithm based on GAC model in image segmentation.

After the number of iterations and running time of the two algorithms were compared, it was found that when the number of iterations was the same, the running time of the improved fuzzy clustering level set algorithm was $11.82 \mathrm{~s}$, while that of the GAC model algorithm was 12.69 s, as shown in Table 1. The running time of the former was $0.87 \mathrm{~s}$ less than that of the latter, indicating that the image segmentation based on the improved fuzzy clustering level set algorithm was better than the GAC model algorithm in running time.

3.3. Evaluation of Segmentation Performance of the Two Methods. The quantitative analysis results of the segmentation performance of the two methods are shown in Table 2. It was noted that the segmentation performance of the improved fuzzy clustering level set algorithm based on MATLAB was better than the classic GAC model algorithm. This proved the effectiveness and feasibility of the improved fuzzy clustering level set algorithm based on MATLAB.

3.4. Comparison of Mean Arterial Pressure. The comparison of the average arterial pressure at six time points of $\mathrm{t} 0, \mathrm{t} 1, \mathrm{t} 2$, $\mathrm{t} 3$, $\mathrm{t} 4$, and $\mathrm{t} 5$ is shown in Figure 4. The average arterial pressure of the experimental group at $\mathrm{t} 0, \mathrm{t} 1, \mathrm{t} 2, \mathrm{t} 3, \mathrm{t} 4$, and $\mathrm{t} 5$ was $112.25 \pm 10.11,101.87 \pm 8.88,99.76 \pm 9.37,97.84 \pm 10.66$, $94.51 \pm 9.42$, and $110.88 \pm 8.46$, respectively. In contrast with the control group, the average arterial pressure of the experimental group at the four time points of $\mathrm{t} 1, \mathrm{t} 2, \mathrm{t} 3$, and $\mathrm{t} 5$ was obviously reduced $(P<0.05)$.

3.5. Comparison of Heart Rate. The comparison of the heart rate at six time points of $\mathrm{t} 0, \mathrm{t} 1, \mathrm{t} 2, \mathrm{t} 3, \mathrm{t} 4$, and $\mathrm{t} 5$ is shown in Figure 5. The heart rates of the experimental group at $t 0, t 1$, $\mathrm{t} 2, \mathrm{t} 3, \mathrm{t} 4$, and $\mathrm{t} 5$ were $78.15 \pm 8.27,73.54 \pm 6.91,69.73 \pm 7.32$, $70.63 \pm 9.66,66.17 \pm 6.77$, and $74.52 \pm 8.01$, respectively. In contrast with the control group, the heart rate of the experimental group at the four time points of $\mathrm{t} 1, \mathrm{t} 2, \mathrm{t} 3$, and $\mathrm{t} 5$ was obviously reduced $(P<0.05)$. 


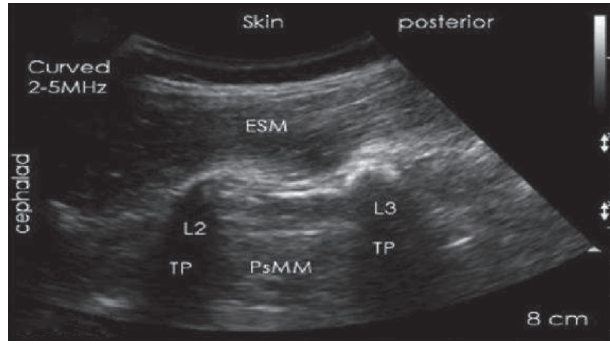

(a)

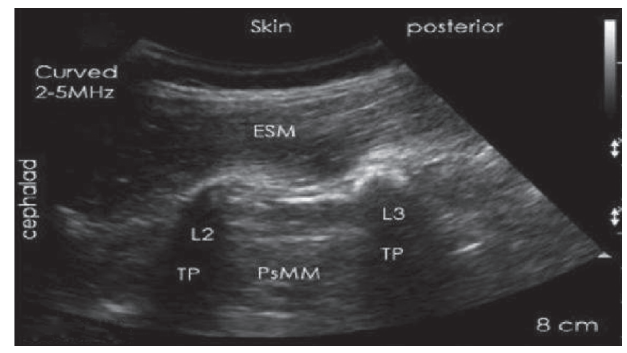

(b)

FiguRE 2: Ultrasonography of the lumbar plexus: before splitting (a) and after splitting (b). ESM meant erector spine muscle, PsMM meant psoas major muscle, and TP meant transverse process.

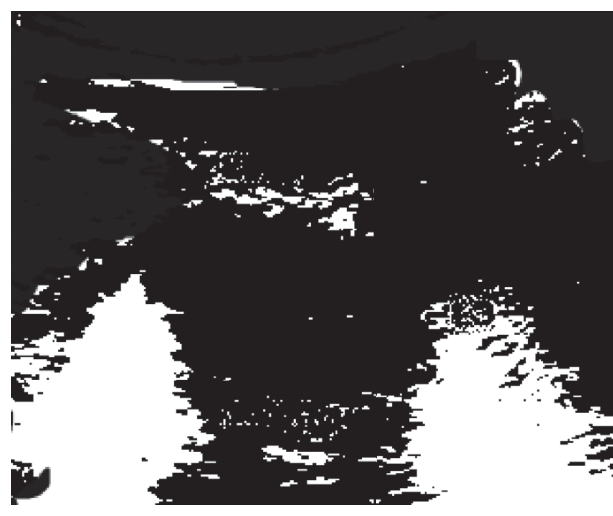

(a)

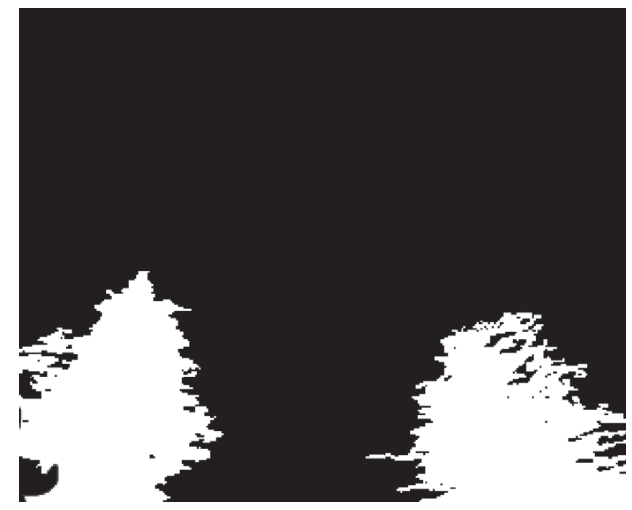

(b)

FIgURE 3: Image segmentation of improved fuzzy clustering level set: clustering results (a) and filtered nontarget areas (b).

TABLe 1: Experimental data.

\begin{tabular}{lcc}
\hline Algorithm & Number of iterations & Running time (s) \\
\hline GAC model algorithm & 800 & 12.69 \\
Improved fuzzy clustering level set algorithm & 800 & 11.82 \\
\hline
\end{tabular}

TABLE 2: Quantitative analysis results of the segmentation performance of the two methods.

\begin{tabular}{|c|c|c|c|}
\hline \multirow{2}{*}{ Segmentation methods } & \multicolumn{3}{|c|}{ Evaluation indicators } \\
\hline & $H_{\max }$ & $U_{r}$ & $C_{r}$ \\
\hline GAC algorithm & 0.877 & 0.915 & 0.676 \\
\hline The improved algorithm & 0.948 & 0.953 & 0.789 \\
\hline
\end{tabular}

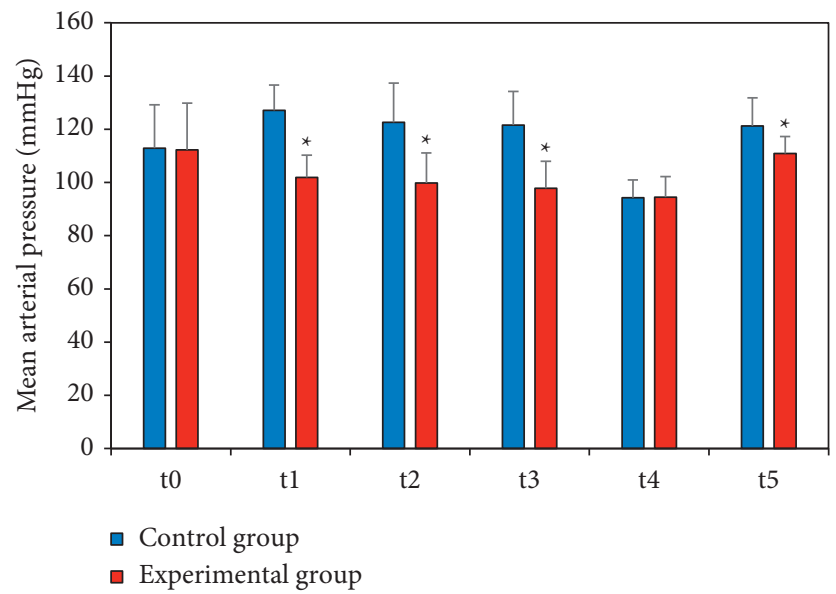

Figure 4: Comparison of mean arterial pressure. ${ }^{*} P<0.05$, in contrast with the control group. 


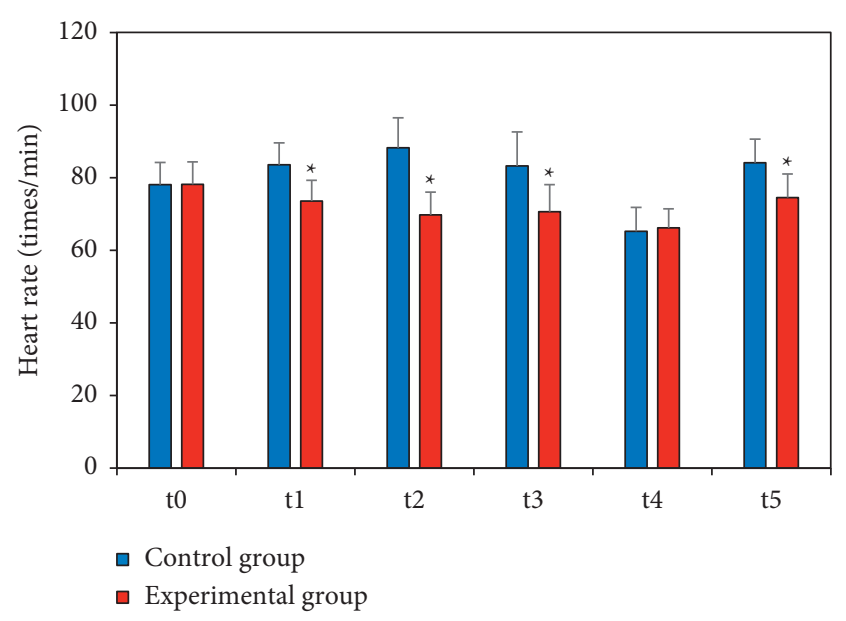

Figure 5: Heart rate comparison. ${ }^{*} P<0.05$, in contrast with the control group.

3.6. Comparison of Intraoperative Sufentanil Dosage and Analgesic Dosage. The comparison results of intraoperative sufentanil dosage and analgesic dosage are shown in Figure 6. The amount of fentanyl and analgesic drugs used in the experimental group was $15.88 \pm 9.26$ and $56.47 \pm 7.45$, respectively; the amount of fentanyl and analgesic drugs used in the control group was $34.76 \pm 7.28$ and $79.76 \pm 9.37$, respectively. In contrast with the control group, the intraoperative sufentanil dosage and analgesic dosage of the experimental group were obviously reduced $(P<0.05)$.

\subsection{Comparison of the Incidence of Postoperative Cognitive} Dysfunction and Delirium. The comparison of the incidence of postoperative cognitive dysfunction and delirium is shown in Figure 7. The incidence of cognitive impairment and delirium in the experimental group was $5.13 \%$ and $2.56 \%$, respectively; the incidence of cognitive impairment and delirium in the control group was $20.51 \%$ and $17.95 \%$, respectively. In contrast with the control group, the incidence of postoperative cognitive dysfunction and delirium in the experimental group was obviously reduced $(P<0.05)$.

\section{Discussion}

In the study, first, a detailed analysis of ultrasound imaging was conducted based on the basis of LSACM to study the impact of different LSACMs on ultrasound imaging. Then, Kumar et al. improved the fuzzy level set image algorithm based on the fuzzy level set image algorithm and the characteristics of the ultrasound image and carried out the segmentation experiment on the lumbar plexus ultrasound image, which was compared with the classic LSACM and GAC model algorithms, and the experimental effects and performance of these two algorithms were checked and analyzed. After experimental analysis, it was found that the improved fuzzy clustering level set algorithm proposed was better than the algorithm based on GAC model in image segmentation. In addition, when the number of iterations was the same, the running time of the improved fuzzy

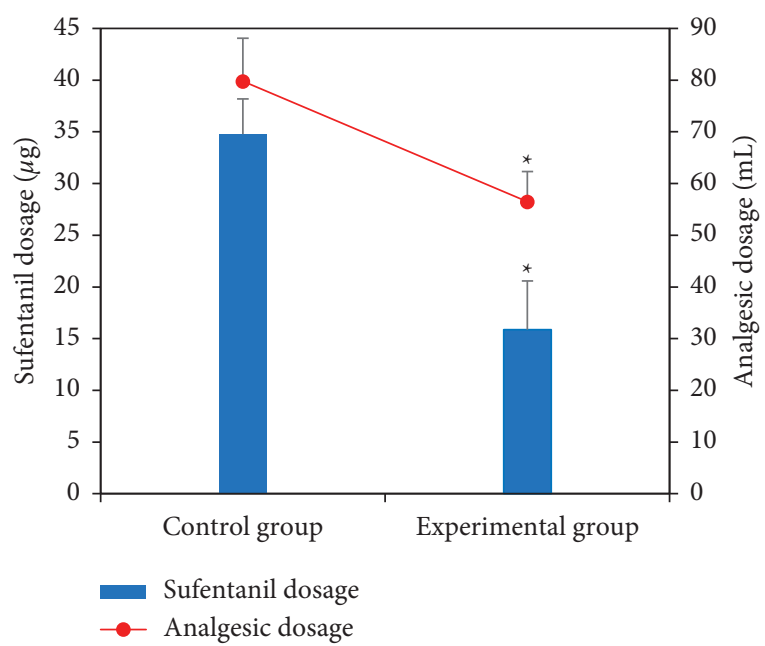

FIgURE 6: Comparison of intraoperative sufentanil dosage and analgesic dosage. ${ }^{*} P<0.05$, in contrast with the control group.

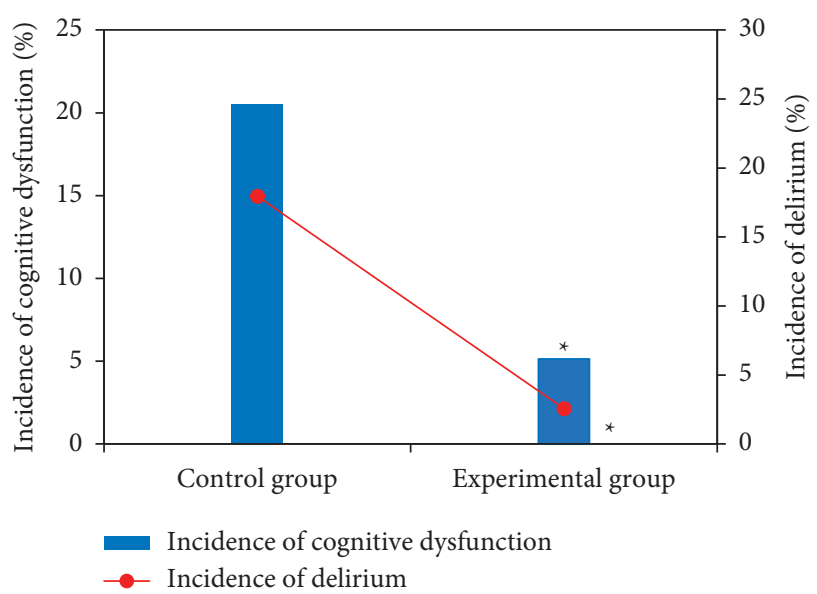

FIGURE 7: Comparison of the incidence of postoperative cognitive dysfunction and delirium. ${ }^{*} P<0.05$, in contrast with the control group.

clustering level set algorithm was $11.82 \mathrm{~s}$, while that of the GAC model algorithm was $12.69 \mathrm{~s}$. The running time of the former was $0.87 \mathrm{~s}$ less than that of the latter, indicating that the image segmentation based on the improved fuzzy clustering level set algorithm was better than the GAC model algorithm in running time, which was similar to the research of Xu et al, who indicated that convolutional neural network had the advantage of wiring harness in breast cancer ultrasound image segmentation, and the accuracy, precision, and recall rate were all over $80 \%$, and the running time was $11.4 \mathrm{~s}$, which was consistent with the results of this study [26], suggesting that these algorithms can improve the imaging quality of medical ultrasound to assist in clinical diagnosis of diseases [27]. Therefore, the improved fuzzy clustering level set algorithm has certain advantages in ultrasound image segmentation and can be recommended for clinical auxiliary diagnosis.

Anesthesia is crucial in the treatment of hip replacement surgery, which can improve the stability of hemodynamics, 
reduce the adverse effects on the patient, and then improve the efficacy of surgery [28]. It was found that in contrast with the control group, the average arterial pressure and heart rate of the experimental group at the four time points of $t$, $\mathrm{t} 2, \mathrm{t} 3$, and $\mathrm{t} 5$ were obviously reduced, and the amount of sufentanil and analgesic drugs and the incidence of postoperative cognitive dysfunction and delirium were obviously reduced, which was consistent with the results of previous studies [29] that general anesthesia is often used for hip fracture surgery, but it often causes adverse reactions during and after the operation and affects the effect of the operation. Peripheral nerve block anesthesia has little effect on patients' breathing and circulation, but traditional nerve block anesthesia often has shortcomings such as inaccurate positioning [30]. The lumbar plexus ultrasound in the study was based on the improved fuzzy clustering level set algorithm, which can realize accurate position to improve the effect of lumbar and sacral plexus block anesthesia. The results suggested that the mean arterial pressure in the experimental group at $\mathrm{t} 0, \mathrm{t} 1, \mathrm{t} 2, \mathrm{t} 3, \mathrm{t} 4$, and $\mathrm{t} 5$ was $112.25 \pm 10.11$, $101.87 \pm 8.88,99.76 \pm 9.37,97.84 \pm 10.66,94.51 \pm 9.42$, and $110.88 \pm 8.46$, respectively; the average arterial pressure at the four time points of $\mathrm{t} 1, \mathrm{t} 2, \mathrm{t} 3$, and $\mathrm{t} 5$ was significantly lower than that of the control group $(P<0.05)$; the heart rates at six time points were $78.15 \pm 8.27,73.54 \pm 6.91$, $69.73 \pm 7.32,70.63 \pm 9.66,66.17 \pm 6.77$, and $74.52 \pm 8.01$, respectively, which were significantly lower than those of the control group $(P<0.05)$; the amounts of fentanyl and analgesics used in the experimental group were 15.88 \pm 9.26 and $56.47 \pm 7.45$, which were lower than $34.76 \pm 7.28$ and $79.76 \pm 9.37$ in the control group $(P<0.05)$; the incidence of cognitive impairment and delirium in the experimental group was $5.13 \%$ and $2.56 \%$, which was lower than $20.51 \%$ and $20.51 \%$ in the control group $(P<0.05)$. In conclusion, combined treatment of hip replacement patients had better heart rate, intraoperative dosage of sufentanil and analgesia, and postoperative incidence of cognitive dysfunction and delirium than patients with general anesthesia alone. Therefore, ultrasound-guided lumbar and sacral plexus block anesthesia combined with general anesthesia has a more perfect anesthesia and analgesic effect, reduces the dosage of drugs, and thereby decreases the occurrence of poor prognosis such as cognitive dysfunction and delirium caused by drugs.

\section{Conclusion}

The level set algorithm based on the improved fuzzy clustering proposed was better than the GAC model algorithm based on the classic level set in image segmentation and running time. Under its guidance, the lumbar and sacral plexus block combined with general anesthesia has better performance in hip arthroplasty, with good clinical application effect, which can reduce the dosage of drugs, thereby decreasing the occurrence of poor prognosis such as cognitive dysfunction and delirium caused by drugs, and is better than simple general anesthesia. However, this study still has some limitations, such as too few algorithm comparisons and small sample size, which will reduce the power of the study. In the follow-up, an expanded sample size is necessary to strengthen the findings of the study. In conclusion, the study can provide a scientific basis for improving the efficiency of ultrasound diagnosis and assisting diagnosis and at the same time promote rehabilitation of hip replacement.

\section{Data Availability}

The data used to support the findings of this study are available from the corresponding author upon request.

\section{Conflicts of Interest}

The authors declare that they have no conflicts of interest.

\section{Acknowledgments}

This study was supported by Medical and Health Science and Technology Project B of Taizhou Science and Technology Bureau (1702KY40).

\section{References}

[1] L. Sahlani, L. Thompson, A. Vira, and A. R. Panchal, "Bedside ultrasound procedures: musculoskeletal and non-musculoskeletal," European Journal of Trauma and Emergency Surgery, vol. 42, no. 2, pp. 127-138, 2016.

[2] U. Tarique, B. Tang, M. Singh, K. M. Kulasegaram, and J. Ailon, "Ultrasound curricula in undergraduate medical education: a scoping review," Journal of Ultrasound in Medicine, vol. 37, no. 1, pp. 69-82, 2018.

[3] A. de Leon, R. Perera, P. Nittayacharn, M. Cooley, O. Jung, and A. A. Exner, "Ultrasound contrast agents and delivery systems in cancer detection and therapy," Advances in Cancer Research, vol. 139, pp. 57-84, 2018.

[4] Q. Huang, Y. Luo, and Q. Zhang, "Breast ultrasound image segmentation: a survey," International Journal of Computer Assisted Radiology and Surgery, vol. 12, no. 3, pp. 493-507, 2017.

[5] D. Mishra, S. Chaudhury, M. Sarkar, and A. S. Soin, "Ultrasound image segmentation: a deeply supervised network with attention to boundaries," IEEE Transactions on Biomedical Engineering, vol. 66, no. 6, pp. 1637-1648, 2019.

[6] H. Park, H. J. Lee, H. G. Kim et al., "Endometrium segmentation on transvaginal ultrasound image using key-point discriminator," Medical Physics, vol. 46, no. 9, pp. 3974-3984, 2019.

[7] P. Gu, W.-M. Lee, M. A. Roubidoux, J. Yuan, X. Wang, and P. L. Carson, "Automated 3D ultrasound image segmentation to aid breast cancer image interpretation," Ultrasonics, vol. 65, pp. 51-58, 2016.

[8] Z. Zhuang, N. Lei, A. N. Joseph Raj, and S. Qiu, "Application of fractal theory and fuzzy enhancement in ultrasound image segmentation," Medical, \& Biological Engineering \& Computing, vol. 57, no. 3, pp. 623-632, 2019.

[9] Y. Li, W. Liang, Y. Zhang, and J Tan, "Automatic global level set approach for lumbar vertebrae CT image segmentation," BioMed Research International, vol. 2018, Article ID 6319879, 12 pages, 2018.

[10] D. R. Ramponi, J. Kaufmann, and G. Drahnak, "Hip fractures," Advanced Emergency Nursing Journal, vol. 40, no. 1, pp. 8-15, 2018. 
[11] B. Rocos, M. R. Whitehouse, and M. B. Kelly, "Resuscitation in hip fractures: a systematic review," BMJ Open, vol. 7, no. 4, Article ID e015906, 2017.

[12] R. H. Quinn, P. A. Mooar, J. N. Murray, R. Pezold, and K. S. Sevarino, "Treatment of hip fractures in the elderly," Journal of the American Academy of Orthopaedic Surgeons, vol. 25, no. 5, pp. e102-e104, 2017.

[13] J. Guay, M. J. Parker, R. Griffiths, and S Kopp, "Peripheral nerve blocks for hip fractures," Cochrane Database of Systematic Reviews, vol. 5, Article ID CD001159, 2017.

[14] S. Kumar, S. K. Ray, and P. Tewari, "A hybrid approach for image segmentation using fuzzy clustering and level set method," International Journal of Image, Graphics and Signal Processing, vol. 4, no. 6, pp. 1-7, 2012.

[15] Y. Xu, Y. Wang, J. Yuan, Q. Cheng, X. Wang, and P. L. Carson, "Medical breast ultrasound image segmentation by machine learning," Ultrasonics, vol. 91, pp. 1-9, 2019.

[16] L. Gui, C. Li, and X. Yang, "Medical image segmentation based on level set and isoperimetric constraint," Physica Medica, vol. 42, pp. 162-173, 2017.

[17] J. Guay, M. J. Parker, R. Griffiths, and S. L. Kopp, "Peripheral nerve blocks for hip fractures," Anesthesia \& Analgesia, vol. 126, no. 5, pp. 1695-1704, 2018.

[18] P. Wennberg, M. Möller, J. Herlitz, and E. Kenne Sarenmalm, "Fascia iliaca compartment block as a preoperative analgesic in elderly patients with hip fractures - effects on cognition," BMC Geriatrics, vol. 19, no. 1, p. 252, 2019.

[19] R. Brull, A. Hadzic, M. A. Reina, and M. J. Barrington, "Pathophysiology and etiology of nerve injury following peripheral nerve blockade," Regional Anesthesia and Pain Medicine, vol. 40, no. 5, pp. 479-490, 2015.

[20] D. Bravo, S. Layera, J. Aliste et al., "Lumbar plexus block versus suprainguinal fascia iliaca block for total hip arthroplasty: a single-blinded, randomized trial," Journal of Clinical Anesthesia, vol. 66, Article ID 109907, 2020.

[21] J. J. Polania Gutierrez, B. Ben-David, C. Rest, M. T. Grajales, and S. K. Khetarpal, "Quadratus lumborum block type 3 versus lumbar plexus block in hip replacement surgery: a randomized, prospective, non-inferiority study," Regional Anesthesia and Pain Medicine, vol. 46, no. 2, pp. 111-117, 2021.

[22] H. Ueshima and H. Otake, "Clinical experiences of pericapsular nerve group (PENG) block for hip surgery," Journal of Clinical Anesthesia, vol. 51, pp. 60-61, 2018.

[23] P. Liu, Y. Wu, Z. Liang, Y. Deng, and Q. Meng, "Comparing the efficacy of pain managements after total hip arthroplasty: a network meta-analysis," Journal of Cellular Biochemistry, vol. 120, no. 3, pp. 4342-4354, 2019.

[24] I. Badiola, J. Liu, S. Huang, J. D. Kelly, and N. Elkassabany, “A comparison of the fascia iliaca block to the lumbar plexus block in providing analgesia following arthroscopic hip surgery: a randomized controlled clinical trial," Journal of Clinical Anesthesia, vol. 49, pp. 26-29, 2018 Sep.

[25] G. Cappelleri, D. Ghisi, A. L. Ambrosoli et al., "Stimulating versus non-stimulating catheter for lumbar plexus continuous infusion after total hip replacement," Minerva Anestesiologica, vol. 85, no. 3, pp. 236-243, 2019 Mar.

[26] J. Li, B. Dong, W. Cai, and W. Gang, "Continuous lumbar plexus block reduces the incidence of early postoperative cognitive dysfunction in elderly patients undergoing hip arthroplasty," Zhong Nan Da Xue Xue Bao Yi Xue Ban, vol. 43, no. 8, pp. 858-863, 2018 Aug 28, in Chinese.

[27] C. E. Darling, S. Y. Pun, T. J. Caruso, and B. C. H. Tsui, "Successful directional thoracic erector spinae plane block after failed lumbar plexus block in hip joint and proximal femur surgery," Journal of Clinical Anesthesia, vol. 49, pp. 1-2, 2018 Sep.

[28] B. Mei, Y. Lu, X. Liu, Y. Zhang, E. Gu, and S. Chen, "Ultrasound-guided lumbar selective nerve root block plus T12 paravertebral and sacral plexus block for hip and knee arthroplasty," Medicine, vol. 98, no. 22, Article ID e15887, 2019.

[29] H. Ueshima, N. Tanaka, and H. Otake, "Greater analgesic effect with intermittent compared with continuous mode of lumbar plexus block for total hip arthroplasty: a randomized controlled trial," Regional Anesthesia and Pain Medicine, vol. 44, no. 6, pp. 632-636, 2019 Jun.

[30] Y. Aoyama, S. Sakura, S. Abe, S. Tadenuma, and Y. Saito, "Continuous quadratus lumborum block and femoral nerve block for total hip arthroplasty: a randomized study," Journal of Anesthesia, vol. 34, no. 3, pp. 413-420, 2020. 